\title{
Survival and prognostic factors in HIV-infected adults treated with Antiretroviral Therapy in a Malaysian referral hospital: A retrospective cohort study
}

\section{Lee Sing Chet}

Universiti Sains Malaysia - Kampus Kesihatan

Siti Azrin Ab Hamid ( $\nabla$ ctazrin@usm.my)

Universiti Sains Malaysia - Kampus Kesihatan

Norsa'adah Bachok

Universiti Sains Malaysia - Kampus Kesihatan

Suresh Kumar Chidambaram

Hospital Sungai Buloh

Research article

Keywords: antiretroviral therapy, human immunodeficiency virus, prognostic, survival

Posted Date: October 4th, 2019

DOl: https://doi.org/10.21203/rs.2.15641/v1

License: (1) (1) This work is licensed under a Creative Commons Attribution 4.0 International License.

Read Full License 


\section{Abstract}

Background: It is well established that antiretroviral therapy (ART) is beneficial in reducing the mortality among patients with human immunodeficiency virus (HIV). In Malaysia, there is lack of study and information regarding the overall survival rates and prognostic factors for survival in HIV-infected adults treated with ART. Therefore, this study aimed to assess and compare the survival rates as well as to identify the prognostic factors for survival among HIV adults in Malaysia.

Methods: A retrospective cohort study was conducted by reviewing the medical records of HIV patients who started ART between year 2007 and 2016 at a tertiary referral hospital in Malaysia. ART-naive adults aged 15 years and above were included and those who were transferred out were excluded. After applying inclusion and exclusion criteria, there were 339 cases eligible in this study. Systematic sampling method was applied. Kaplan Meier survival curve and log-rank test were used to compare the overall survival rates. Cox proportional hazards regression was applied to determine the prognostic factors for survival.

Results: The estimated overall survival rates were $95.9 \%, 93.8 \%, 90.4 \%, 84.9 \%$, and $72.8 \%$ at 6 months, 1 year, 3 years, 5 years and 10 years, respectively. The overall survival rates were significantly different according to age group ( $p<0.001)$, employment status $(p<0.001)$, transmission mode $(p=0.003)$, and history of illicit drug use $(p=0.017)$, baseline CD4 cell count $(p<0.001)$, baseline haemoglobin level $(p<0.001)$, tuberculosis co-infection $(p<0.001)$, hepatitis co-infection $(p=0.008)$, first NRTI $(p<0.001)$ and history of defaults $(p=0.021)$. Based on multiple Cox regression, patients who were anaemic had 3.76 times $(95 \% \mathrm{Cl}: 1.97,7.18 ; p<0.001)$ higher hazard of death than their non-anaemic counterparts. The hazard risk was 2.09 times ( $95 \% \mathrm{Cl}$ : 1.10, 3.96; $\mathrm{p}=0.024)$ higher among HIV patients co-infected with tuberculosis compared to those who were not.

Conclusion: Overall survival rates were higher than low-income countries but lower than in high-income countries, and comparable with middle-income countries. Low baseline haemoglobin level and tuberculosis co-infection were strong prognostic factors for HIV survival

\section{Background}

Since the first cases of acquired immunodeficiency syndrome (AIDS) reported in 1981, more than 77 million people have acquired human immunodeficiency virus (HIV) globally and 35 million have died of HIV-related illnesses [1]. In year 2017, there were estimated 36.9 million people living with HIV (PLHIV) worldwide. A total of $2.5 \%$ of them (940000) died due to HIV/AIDS. However, following global efforts in scaling up the antiretroviral treatment coverage, the HIV-related death rate had seen a 50.5\% decline from a peak of 1.9 million in 2005 to 940,000 thousand in year 2017 [2]. 
Comparatively, in Malaysia, there was an estimated 87,000 PLHIV in year 2017 of which 4,400 deaths (5.1\%) were related to HIV/AIDS - a higher death rate compared to global data. The mortality rate was also on a decreasing trend but at a much slower rate of $13.7 \%$ for the same 12-year period. The total number of HIV/AIDS deaths in Malaysia reduced from 5,100 in 2005 to 4,400 in year 2017 [2].

In year 1997, antiretroviral therapy (ART) was introduced in Malaysia but was not widely used due to its cost. Selected patients were only subsidised one antiretroviral drug if they fulfilled the selection criteria. The ART provision policy was then upgraded to two-drug subsidy policy in 2004. The Malaysian government had continually updated its policy to expand the availability and accessibility of ART to its people. In 2006, first-line ART was provided for free to all PLHIV at government hospitals and clinic. In 2010, the policymakers continued their effort to reach more PLHIV by shifting the threshold of starting ART from CD4 level of 200 cells/ $\mu \mathrm{L}$ to 350 cells/ $\mu \mathrm{L}$ [3].

Even though it is well established that ART is beneficial in reducing mortality, the extent of its benefit varies across regions [4]. In Malaysia, there is a lack of information regarding prognostic factors for survival in HIV-infected adults treated with ART. The local published studies conducted to determine the mortality rate and its determinants in HIVinfected patients are limited. Moreover, these local studies covered a wide range of time from as early as 1987 to 2009 during which time, the antiretroviral drug availability and its provision policy had changed significantly in Malaysia [5, 6]. Therefore, this study aimed to determine the overall survival rates and to identify the prognostic factors for survival in HIV-infected adults in Malaysia.

\section{Methods}

\section{Study Design and Population}

This retrospective cohort study was conducted among all HIV-infected adults registered in Hospital Sungai Buloh, Malaysia between 1 January 2007 and 31 December 2016. This hospital was chosen as the study site as it is considered as one of the centres of excellence 
for infectious disease in Malaysia. Besides, the hospital is in Selangor state which had reported the highest number of PLHIV in Malaysia.

Only patients aged above 15 years old and who were ART-naive were included in the study. Patients who were transferred out from the hospital were excluded. After applying the inclusion and exclusion criteria, a total of 339 cases were eligible in this study.

The sample size was calculated based on survival formula using the Power and Sample Size Calculations (PS) Software [7] with the level of significance of 0.05 and $80 \%$ power of the study. The accrual time during which patients were recruited was 120 months and the additional follow-up time after the end of recruitment was 20 months. Systematic sampling was applied for this study to select the study sample.

\section{Data Collection}

Data collection form was used to record all required information from the electronic medical records in the e-Hospital Information System (e-HIS) database of Hospital Sungai Buloh. Review of the electronic medical records was carried out at the Infectious Disease Clinic of the hospital. The required information was retrieved from the electronic medical record for each patient one at a time before being transferred manually to the data collection form.

The primary outcome of interest in this study was death from all causes. For those who were lost to follow-up during the study period or still alive at study closure, the observations were censored. A patient was defined as lost to follow-up when he or she defaulted the scheduled follow-up and did not turn up at the clinic within three months after the appointment date. If a patient turned up beyond three months after the appointment date, he or she would be considered as having a history of defaulting follow-up. For these patients, the final status before study closure would be recorded as the outcome for the analysis.

Death status was verified by matching the patients' records with the database of the National Registration Department. The patients' information including their full name and the National Registration Identity Card number were used for the matching purpose. 
Study variables were patient-related, clinical-related, and treatment-related. The patient-related characteristics were: age; bodyweight; gender; ethnicity; employment status; mode of transmission; history of illicit drug use; and smoking status. The clinical-related characteristics were: baseline CD4 cell count; baseline viral load; baseline haemoglobin level; WHO clinical staging; tuberculosis (TB) coinfection; hepatitis B co-infection; hepatitis C co-infection; opportunistic infection; and duration of first HIV positive test to ART initiation.

As for treatment-related characteristics, the variables included year of ART initiation; first nonnucleoside reverse transcriptase (NRTI) background; and history of defaults. Anaemia was defined as haemoglobin level less than $11 \mathrm{~g} / \mathrm{dL}$ based on expert opinion and previous studies $[8,9]$.

\section{Statistical Analysis}

Data analyses were performed using STATA SE Version 14. Categorical data was presented in frequency (n) and percentage (\%). For numerical data, it was described in mean and standard deviation (SD) if it was normally distributed. Alternatively, the median and interquartile range (IQR) was reported for nonnormally distributed data.

Survival time was defined as the duration from the date of ART initiation to the date of the event. For patients who were lost to follow-up, the follow-up time was censored on the date of the most recent follow-up visits. Patients who were still alive at study closure were censored on 31 August 2018.

The overall survival rates were estimated using Kaplan-Meier survival curve. The log-rank tests were used to compare the survival curves. In the event where there were multiple pairwise comparisons, Bonferroni correction was made by multiplying the p-value with the number of comparisons. Cox proportional hazards regression was conducted to determine the prognostic factors for HIV survival.

Results were presented as a crude and adjusted hazard ratio (HR) and 95\% confidential interval (Cl); a p-value $<0.05$ was regarded as statistically significant. 


\section{Results}

\section{Socio-Demographics of Patients}

The mean age of patients during ART initiation was 37.0 (11.2) years old with majority of patients were male (85.3\%). Malay ethnicity constituted $43.4 \%$ of all patients. Sexual transmission was the commonest HIV transmission mode reported. Sixty-one (18.0\%) patients had a history of illicit drug use such as heroin and methamphetamine as summarised in Table 1.

\section{Clinical- And Treatment-Related Characteristics}

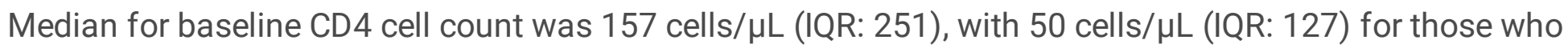
died and 192 cells/ $\mu \mathrm{L}$ (IQR: 248) for the censored patients. Based on the baseline haemoglobin level, there were 78 (25.2\%) patients who were anaemic, with a mean of $12.7 \mathrm{~g} / \mathrm{dL}$ (SD: 2.4 ).

A total of 85 patients $(25.1 \%), 27$ patients ( $8.0 \%)$ and 50 patients $(14.7 \%)$ were diagnosed withTB, hepatitis $B$ and hepatitis $C$ co-infection, respectively. Two of them $(0.6 \%)$ were co-infected with both hepatitis B and C co-infection. Throughout the study period, less than half $(47.5 \%)$ of the patients had experienced at least one episode of opportunistic infection. Of it, the most common opportunistic infections were candidiasis (21.9\%), Pneumocystis jiroveci pneumonia (19.7\%), and herpes infection (15.3\%) as tabulated in Table 2.

The overall median for the duration from the first HIV positive test to ART initiation was 3.5 months (IQR: 20.8). A total of 63 patients (18.6\%) had history of defaulting follow-up during the study period. 


\section{Survival of HIV-infected adults treated with ART}

Overall, there were 50 deaths (14.7\%) and 289 censored events (85.3\%). Among 50 patients who died, the majority (42.0\%) died within the first year of ART initiation, 14 patients $(28.0 \%)$ died during first six months of ART initiation and seven patients (14.0\%) died between six and 12 months of ART initiation. Of the censored observation, 241 patients (83.4\%) were alive at the end of the study period and 48 patients (16.6\%) were lost to follow-up during the study period. The overall median follow-up time of all the patients was $4.3(4.1)$ years.

The overall survival rates at 6 months, one year, three years, five years and ten years were $95.9 \%$, $93.8 \%, 90.4 \%, 84.9 \%$ and $72.8 \%$, respectively. During the 1,600 person-years of observation, the mortality rate was $3.12(95 \% \mathrm{Cl}: 2.37,4.12)$ per 100 person-years.

Based on the log-rank test, the overall survival rates were significantly different for age group $(p<0.001)$, employment status $(p<0.001)$, HIV transmission mode $(p=0.003)$, and history of illicit drug use $(p=0.017)$. The overall survival rates were lowest among those whose age were 50 years old and above, unemployed, infected via injection drug use, and had a history of illicit drug use (Figure 1).

The overall survival rates for the clinical-related variables were significantly different including baseline CD4 cell count $(p<0.001)$, baseline haemoglobin level $(p<0.001)$, TB $(\mathrm{p}<0.001)$, and hepatitis $\mathrm{C}(\mathrm{p}=0.008)$. The baseline CD4 cell count was inversely associated with overall survival rates. Those whom baseline CD4 cell count was $0-49$ cells $/ \mu \mathrm{L}$ and 50199 cells/pL had lower overall survival rates (70.6\%; 95\% CI: 58.0, 80.1 and 63.6\%; 95\% CI: 39.6, 80.1, respectively) compared to those with baseline CD4 cell count of 200 cells/ $\mu \mathrm{L}$ or above (89.6\%; 95\% CI: 77.3, 95.4). Patients who were anaemic at baseline, co-infected with TB and hepatitis C had lower overall survival rates (Figure 2). 
With regards to treatment-related characteristics, first NRTI background $(p<0.001)$ and history of defaults $(\mathrm{p}=0.021)$ had significant differences in overall survival rates. Patients started with the combination of stavudine and lamivudine as the first ART background and those who had a history of defaults had lower overall survival rates (Figure 3).

\section{Prognostic Factors of HIV-infected adults treated with ART}

Age group, bodyweight, employment status, HIV transmission mode, history of illicit drug use, baseline CD4 cell count, baseline haemoglobin level, co-infection of TB and hepatitis C. year of ART initiation, first NRTI background and history of defaults were significantly associated with the overall survival of HIV patients using simple Cox proportional hazards regression.

In multivariable analysis, baseline haemoglobin level and tuberculosis co-infection, were found to be significant prognostic factors for HIV survival. HIV patients who were anaemic had 3.76 times increased hazards of dying compared to their non-anaemic counterparts. The hazard risk was 2.09 times higher among HIV patients who were co-infected with TB than those who were not (Table 3).

\section{Discussion}

The survival rates of HIV-infected adults treated with ART in this current study were lower compared to England and Wales [10,11] and higher compared to Tanzania, Nepal and Ethiopia [12-14]. Comparing with China, a middle-income country, the survival rates in this study were lower in the first year of ART but became comparable with longer time on ART [15].

When a comparison with the previous Malaysian study was made, the current study had higher survival rates. This could be due to the use of ART in all patients in the current study, whereas the previous study included patients started with single- or double- or triple-drug regimens [5]. Another reason for low survival rates compared to other Malaysian study probably because the patients who were started ART were chosen based on specific selection criteria due to limited government subsidy prior to 2006 [6].

Further analysis was performed and found that 14 patients in this study who died within the first six months on ART had poor baseline characteristics. They were consisted of a higher percentage of 
patients with age older than 50 years old (six out of 14), anaemic (ten out of 14), low baseline CD4 cell count which was less than 50 cells/ $\mu \mathrm{L}$ (11 out of 14) and had tuberculosis (eight out of 14 ), when compared to the entire study population. The similar was explained in a China study that poor baseline patient characteristics contributed largely to the worse survival. The authors suggested that early diagnosis and treatment within the first six months of ART were important for improving survival outcome in HIV-infected patients [16].

Anaemia, which was based on the baseline haemoglobin level, is a well-recognised predictor for survival among HIV patients. The result of this study was consistent with previous studies. An Ethiopian study reported that HIV patients with baseline haemoglobin level less than $11 \mathrm{~g} / \mathrm{dL}$ had an increased hazard risk of 3.06 compared to their counterparts [8]. In Tanzania, moderate and severe anaemia were reported to increase the hazard risk by 7.50 and 9.20 times, respectively [12]. Neighbouring country Vietnam somehow reported a lower hazard ratio. HIV patients with lower baseline haemoglobin level of less than $10 \mathrm{~g} / \mathrm{dL}$ had 1.9 times higher risk to die compared to those whom haemoglobin level was 10 $\mathrm{g} / \mathrm{dL}$ and above [17]. A previous study in Malaysia showed the same inverse relationship between baseline haemoglobin level and mortality. The authors analysed baseline haemoglobin level as a continuous variable and concluded that the hazard risk decreased by $16 \%$ (with every increase of haemoglobin level by $1 \mathrm{~g} / \mathrm{dL}$ [6].

Baseline haemoglobin level was not only predictive of long-term mortality, it was also significantly associated with early mortality during the first six months of ART. In the same study, for long term mortality risk beyond six months of ART, having persistent anaemia increased the risk by 4.9 times compared to no anaemia [18].

Although anaemia was known to increase mortality risk, the possibility of reverse causality should be considered. Anaemia at baseline could be due to HIV disease itself, particularly when the disease was at its advanced stage. Being a common haematologic complication in HIV-infected patients, the aetiologies of anaemia were multifactorial. Anaemia could occur due to bone marrow suppression, micronutrient deficiency, impaired erythropoiesis, systematic fungal and mycobacterial infection, malignancy, or association with chronic disease [19]. 
Anaemia which occurs as a result of co-infection may render a longer recovery time or lead to a poor prognosis of recovery. Thus, these patients have a higher mortality risk [17]. Fortunately, anaemia could be improved with ART initiation, but certain antiretroviral drugs like zidovudine was known to cause bone marrow toxicity leading to anaemia [20]. Therefore, it is important to monitor haemoglobin level periodically according to the national guidelines if zidovudine was the choice of drug in the ART regimen.

Another prognostic factor for HIV survival in this study was TB co-infection. The result of the current study was comparable with previous studies in countries across different regions including China, Ethiopia, Myanmar, and Iran [15, 21, 22, 23].

A Malaysian study involving HIV/TB patients looked further into the association of types of tuberculosis with mortality. When compared to extrapulmonary TB both smear positive pulmonary TB and smear negative pulmonary TB were not prognostic for a higher mortality [24]. The same result was reported in a study in Myanmar reporting that pulmonary TB was not found to be a predictor for death based on multiple Cox proportional hazards regression [22].

HIV and TB speed up each other's disease progression, thus forming a lethal combination. TB is the leading cause of death among HIV patients $[25,26]$. When it involves HIV patients co-infected with TB, the management becomes complicated because of the known drug-drug interactions between the drug used in HIV and TB treatment.

Antiretroviral drugs such as non-nucleoside reverse transcriptase inhibitors (NNRTIs) and protease inhibitors (PIs) are inducers or inhibitors of drug metabolising enzymes and anti-TB such as rifampicin is a potent inducer of metabolising enzymes and transporters. The consequences of the interactions include therapeutic failure and increased toxicity, which in turn could increase the mortality risk amongst HIV/TB co-infected patients. However, with the systematic approach in identifying and managing individual drug regimens, mortality rates in this group of patients could be reduced. WHO recommends starting ART in all HIV/TB co-infected patients regardless of their CD4 cell count. Being the most common co-infection among HIV patients, it is therefore important to collaborate HIV and TB care services for this group of patients $[27,28]$. 
It was expected that CD4 cell count would be a significant predictor for HIV survival in this current study, but the results of multiple Cox regression showed the otherwise. This result could be explained by the small proportion of patients within the event group in this current study. A study in India showed that lower adherence to ART was observed among those with CD4 cell count less than 200 cells/ $\mu \mathrm{L}$, which could explain the higher mortality rates among those with low CD4 cell count [29]. However, adherence was not considered in this study which had also been a limitation of this study.

\section{Strength and Limitation}

This study was conducted at one of the biggest tertiary hospital under Ministry of Health Malaysia. Hence, the results can be applied to other hospitals in Malaysia as all under the Ministry of Health share the same system and support. All patients included in this study were treatment naïve, therefore the results are not confounded by previous ART. The mortality data was from reliable sources, i.e. the National Registration Department, adding to the quality of the current study.

Due to the retrospective design of the study, there was a high percentage of missing data for some of the variables of interest which were then excluded from survival analysis, such as baseline viral load and WHO clinical staging. This could cause bias in the assessment of progression to mortality. Besides, the current study did not include the factor regarding adherence to ART, which may have caused bias in identifying prognostic factors for HIV survival. This information was not included as it was not consistently recorded in medical records. Neither was the assessment of ART adherence standardised among different healthcare workers.

\section{Conclusion And Recommendation}

The current study has shown that the overall survival rates among HIV patients in Hospital Sungai Buloh is higher than low-income countries but lower than high-income countries, and comparable with other middle-income countries. Most of the deaths occurred within the first year of ART initiation. This implies 
the importance of early treatment for HIV-infected patients especially those who are presented with low haemoglobin level or have TB co-infection at presentation to care.

The results of this study could be used to identify patients who should be paid extra attention to link them to HIV care. These patients are those newly tested HIV positive patients who have low haemoglobin level and do not present with any symptoms at the first clinic visit. Being asymptomatic and feeling healthy, these patients may be reluctant to start ART because they do not perceive the benefits of ART in sustaining their health. Moreover, the concept of lifelong therapy for HIV treatment may hinder them from entering into care [30]. The results of the current study regarding the increased mortality with low baseline haemoglobin level could be used by the clinicians to convince this group of patients to be engaged and remain in care.

This study results also suggest for prioritisation of care for HIV patients who are co-infected with TB. The clinicians could share the results of this study to their patients alongside extensive counselling on the importance of being compliant to both HIV and TB treatment. The results also reinforce the importance of offering six-month isoniazid prophylaxis therapy (IPT) to all HIV patients to reduce overall TB risk after active tuberculosis has been ruled out. This group of patients should be informed of the higher mortality risk associated with TB co-infection, so that they comply with the IPT.

\section{Abbreviations}

AIDS: Acquired immunodeficiency syndrome; ART: Antiretroviral therapy; CI: Confidence interval; HIV: Human immunodeficiency virus; HR: Hazard ratio; IQR: Interquartile range; NRTI: non-nucleoside reverse transcriptase; SD: Standard deviation; TB: Tuberculosis: WHO: World Health Organization

\section{Declarations}


This research study had ethical approval from the National Medical Research and Ethics Committee (MREC) of the Ministry of Health Malaysia via the National Medical Research Registry (NMRR) (Ref: KKM.NIHSEC. P18-1703(6) and NMRR-18-2100-42257(IIR)). Besides, approval had also been obtained from the Research Ethics Committee of Universiti Sains Malaysia (Ref: USM/JEPeM/18060287).

Consent was not required in this study since it involved retrospective cohort study where the researcher only need to review the medical records of the patients.

All data were anonymous, and the data collected did not contain unique identifier. Data was entered into analysis software in a password-protected computer with limited accessibility. All data was kept strictly confidential.

\section{Consent for publication}

Not applicable

\section{Availability of data and materials}

The authors declare that no data have been fabricated or manipulated (including images) to support the conclusions. The authors declare that no data, text, or theories by others are presented as if they were the authors own.

\section{Competing interest}

The author(s) declared no potential conflicts of interest with respect to the research, authorship and/or publication of this article.

\section{Funding}


This research was funded by Universiti Sains Malaysia Bridging Grant 2018 with grant number: 304.PPSP.6316327.

\section{Authors' contributions}

LSC, SAAH, NB and SKC performed the conceptualization of the study; LSC, SAAH, NB performed the analysis; SAAH performed the funding acquisition; LSC, SAAH, NB and SKC performed the methods of the study; SAAH, NB and SKC supervised the study; LSC wrote the original draft, SAAH, NB and SKC reviewed \& edited the manuscript. All authors read and approved the final manuscript.

\section{Acknowledgements}

The authors acknowledge the Universiti Sains Malaysia for supporting this study. We would like to thank the Director General of Health Malaysia for his permission to publish this article. We are also grateful to the Unit of Infectious Disease, Hospital Sungai Buloh for the administrative and technical support during the data collection process.

\section{References}

1. Global HIV and AIDS statistics 2018 factsheet. Available from: https://www.unaids.org/en/resources/fact-sheet [Accessed 5 May 2019]

2. AIDSinfo. Available from: https://aidsinfo.unaids.org/ [Accessed 5 May 2019]

3. Ministry of Health Malaysia. Ending AIDS in Malaysia: myth or reality? : Ministry of Health Malaysia, HIV/STI Sector; 2015.

4. Biset Ayalew M. Mortality and its predictors among HIV infected patients taking antiretroviral treatment in Ethiopia: a systematic review. AIDS Res Treatment. $2017 ; 2017$.

5. Lubis R, Bulgiba A, Kamarulzaman A, Hairi NN, Dahlui M, Peramalah D. Predictors of death in Malaysian HIV-infected patients on anti-retroviral therapy. Prev Med. 2013;57 Suppl:S54-6. 
6. Mat Shah R, Bulgiba A, Lee CKC, Haniff J, Mohamad Ali M. Highly active antiretroviral therapy reduces mortality and morbidity in patients with AIDS in Sungai Buloh Hospital. J Exp Clin Med. 2012;4(4):239-44.

7. Dupont WD, Plummer Jr WD. PS power and sample size program available for free on the Internet. Control Clin Trials. 1997;18(3):274.

8. Belay H, Alemseged F, Angesom T, Hintsa S, Abay M. Effect of late HIV diagnosis on HIV-related mortality among adults in general hospitals of Central Zone Tigray, northern Ethiopia: a retrospective cohort study. HIV AIDS (Auckl). 2017;9:187-92.

9. Tadesse, K., Haile, F., \& Hiruy, N. Predictors of mortality among patients enrolled on antiretroviral therapy in Aksum hospital, northern Ethiopia: a retrospective cohort study. PLoS One.2004;9(1), e87392.

10. Lesko CR, Cole SR, Miller WC, Westreich D, Eron JJ, Adimora AA, et al. Ten-year survival by race/ethnicity and sex among treated, HIV-infected adults in the United States. Clin Infect Dis. 2015;60(11):1700-7.

11. Croxford S, Kitching A, Desai S, Kall M, Edelstein M, Skingsley A, et al. Mortality and causes of death in people diagnosed with HIV in the era of highly active antiretroviral therapy compared with the general population: an analysis of a national observational cohort. Lancet Public Health. 2017;2(1):e35-e46.

12. Johannessen A, Naman E, Ngowi BJ, Sandvik L, Matee MI, Aglen HE, et al. Predictors of mortality in HIV-infected patients starting antiretroviral therapy in a rural hospital in Tanzania. BMC Infect Dis. 2008;8:52.

13. Bhatta L, Klouman E, Deuba K, Shrestha R, Karki DK, Ekstrom AM, et al. Survival on antiretroviral treatment among adult HIV-infected patients in Nepal: a retrospective cohort study in far-western Region, 2006-2011. BMC Infect Dis 2013;13(1):604.

14. Damtew B, Mengistie B, Alemayehu T. Survival and determinants of mortality in adult HIV/Aids patients initiating antiretroviral therapy in Somali Region, Eastern Ethiopia. Pan Afr Med J. 2015;22:138.

15. Zhang G, Gong Y, Wang Q, Deng L, Zhang S, Liao Q, et al. Outcomes and factors associated with survival of patients with HIV/AIDS initiating antiretroviral treatment in 
Liangshan Prefecture, southwest of China: A retrospective cohort study from 2005 to 2013. Med(Baltimore). 2016;95(27):e3969.

16. Ren L, Li J, Zhou S, Xia X, Xie Z, Liu P, et al. Prognosis of HIV Patients Receiving Antiretroviral Therapy According to CD4 Counts: A Long-term Follow-up study in Yunnan, China. Sci Rep. 2017;7(1):9595.

17. Cuong do D, Thorson A, Sonnerborg A, Hoa NP, Chuc NT, Phuc HD, et al. Survival and causes of death among HIV-infected patients starting antiretroviral therapy in northeastern Vietnam. Scand J Infect Dis. 2012;44(3):201-8.

18. Fregonese F, Collins IJ, Jourdain G, LeCoeur S, Cressey TR, Ngo-Giang-Houng N, et al. Predictors of 5-year mortality in HIV-infected adults starting highly active antiretroviral therapy in Thailand. J Acquir Immune Defic Syndr. 2012;60(1):91-8.

19. Pande A, Bhattacharyya M, Pain S, Ghosh B, Saha S, Ghosh A, et al. Anemia in antiretroviral naive HIV/AIDS patients: a study from Eastern India. Online J Health Allied Sci. 2012;10(4 (4)).

20. Gedefaw L, Yemane T, Sahlemariam Z, Yilma D. Anemia and risk factors in HAART naive and HAART experienced HIV positive persons in south west Ethiopia: a comparative study. PLoS One. 2013;8(8):e72202.

21. Tachbele E, Ameni G. Survival and predictors of mortality among human immunodeficiency virus patients on anti-retroviral treatment at Jinka Hospital, South Omo, Ethiopia: a six years retrospective cohort study. Epidemiol Health. 2016;38.

22. Aung ZZ, Saw YM, Saw TN, Oo N, Aye HNN, Aung S, et al. Survival rate and mortality risk factors among TB-HIV co-infected patients at an HIV-specialist hospital in Myanmar: A 12-year retrospective follow-up study. Int J Infect Dis. 2019;80:10-5.

23. Mirzaei M, Poorolajal J, Khazaei S, Saatchi M. Survival rate of AIDS disease and mortality in HIV-infected patients in Hamadan, Iran: a registry-based retrospective cohort study (1997-2011). Int J STD \& AIDS. 2013;24(11):859-66.

24. Ismail I, Bulgiba A. Predictors of death during tuberculosis treatment in TB/HIV coinfected patients in Malaysia. PLoS One. 2013;8(8):e73250. 
25. Bhowmik A, Bhandari S, De R, Guha SK. Predictors of mortality among HIV-infected patients initiating anti retroviral therapy at a tertiary care hospital in Eastern India. Asian Pac J Trop Med. 2012;5(12):986-90.

26. Lee SH, Kim K-H, Lee SG, Cho H, Chen DH, Chung JS, et al. Causes of death and risk factors for mortality among HIV-infected patients receiving antiretroviral therapy in Korea. J Korean Med Sci. 2013;28(7):990-7.

27. World Health Organization. Consolidated guidelines on the use of antiretroviral drugs for treating and preventing HIV infection. 2016.

28. Ministry of Health Malaysia. Malaysian Consensus Guidelines on Antiretroviral Therapy. 2017.

29. Rai S, Mahapatra B, Sircar S, Raj PY, Venkatesh S, Shaukat M, et al. Adherence to antiretroviral therapy and its effect on survival of HIV-infected individuals in Jharkhand, India. PLoS One. 2013;8(6):e66860.

30. Tweya H, Oboho IK, Gugsa ST, Phiri S, Rambiki E, Banda R, et al. Loss to follow-up before and after initiation of antiretroviral therapy in HIV facilities in Lilongwe, Malawi. PLoS One. 2018;13(1):e0188488.

\section{Tables}

Table 1 Socio-demographics of HIV-infected adults treated with ART in Hospital Sungai Buloh ( $n=339)$. 


\begin{tabular}{|c|c|c|c|}
\hline \multirow[t]{2}{*}{ Variables } & \multicolumn{3}{|c|}{ Frequency, n (\%) } \\
\hline & Death $(n=50)$ & Censored $(n=289)$ & Total $(n=339)$ \\
\hline \multicolumn{4}{|l|}{ Age (years) } \\
\hline$<30$ & $7(7.3)$ & 89 (92.7) & $96(28.3)$ \\
\hline $30-39$ & $13(10.6)$ & $110(89.4)$ & $123(36.3)$ \\
\hline $40-49$ & $16(20.5)$ & $62(79.5)$ & $78(23.0)$ \\
\hline$\geq 50$ & $14(33.3)$ & $28(66.7)$ & $42(12.4)$ \\
\hline \multicolumn{4}{|l|}{ Gender } \\
\hline Male & $42(14.5)$ & $247(85.5)$ & $289(85.3)$ \\
\hline Female & $8(16.0)$ & $42(84.0)$ & $50(14.7)$ \\
\hline \multicolumn{4}{|l|}{ Ethnicity } \\
\hline Malay & $23(15.7)$ & $124(84.4)$ & $147(43.4)$ \\
\hline Chinese & $17(13.4)$ & $110(86.6)$ & $127(37.5)$ \\
\hline Others & $10(15.4)$ & $55(84.6)$ & $65(19.2)$ \\
\hline \multicolumn{4}{|l|}{ Mode of transmission } \\
\hline Homosexual/ bisexual & $8(6.0)$ & $126(94.0)$ & $134(39.5)$ \\
\hline Heterosexual & $26(19.1)$ & $110(80.9)$ & $136(40.1)$ \\
\hline Injection drug use & $13(28.9)$ & $32(71.1)$ & $45(13.3)$ \\
\hline Unknown & $3(12.5)$ & $21(87.5)$ & $24(7.1)$ \\
\hline \multicolumn{4}{|l|}{ History of illicit drug use } \\
\hline No & $21(9.8)$ & $193(90.2)$ & $214(63.1)$ \\
\hline Yes & $14(23.0)$ & $47(77.1)$ & $61(18.0)$ \\
\hline Unknown & $15(23.4)$ & $49(76.6)$ & $64(18.9)$ \\
\hline
\end{tabular}

Table 2 Clinical- and treatment-related characteristics of HIV-infected adults treated with ART in Hospital Sungai Buloh ( $\mathrm{n}=339)$. 


\begin{tabular}{ccc}
\hline Death & Censored & Total \\
$(n=50)$ & $(n=289)$ & $(n=339)$ \\
\hline
\end{tabular}

\section{Baseline CD4 cell count (cells/ $\mu \mathrm{L}$ )}

$\geq 200$

$8(5.6)$

$134(94.4)$

142

50-199

16 (18.0)

$73(82.0)$

89 (26.3)

$0-49$

$23(24.0)$

73 (76.0)

96 (28.3)

Unknown

$3(25.0)$

$9(75.0)$

$12(3.5)$

Baseline viral load (copies/ml)

$<10,000$

$4(11.4)$

31 (88.6)

35 (10.3)

$10,000-100,000$

4 (5.6)

67 (94.4)

71 (20.9)

$\geq 100,000$

17 (16.4)

87 (83.7)

104

(30.7)

Unknown

$25(19.4)$

$104(80.6)$ $129(38.1)$

Baseline haemoglobin level ( $\mathrm{g} / \mathrm{dL}$ )

No anaemia

$17(7.4) \quad 214(92.6)$

231

(68.1)

Anaemia

24 (30.8)

54 (69.2)

78 (23.0)

Unknown

9 (30.0)

$21(70.0)$

$30(8.9)$

\section{WHO clinical staging}

Class I

$7(6.2)$

106 (93.8)

Class II

$2(8.0)$

23 (92.0)

25 (7.4)

Class III

4 (12.9) 27 (87.1)

31 (9.1)

Class IV

1 (6.7)

14 (93.3)

$15(4.4)$

Unknown

$36(23.2) \quad 119(76.8)$

155

(45.7)

\section{Tuberculosis}

No

$25(9.8) \quad 229(90.2)$ 
Yes

\section{Hepatitis B}

No

Yes

Hepatitis C

No

Yes

Opportunistic infection

No

Yes

Duration of first HIV positive test to ART initiation (days) ${ }^{*}$

Year of ART initiation

2007-2010

2011-2013

2014-2016

\section{First NRTI background}

TDF/ FTC

ZDV/ 3TC

$\mathrm{d} 4 \mathrm{~T} / 3 \mathrm{TC}$

$\mathrm{ABC} / 3 \mathrm{TC}$
$25(29.4) \quad 60(70.6)$

$85(25.1)$
312

$4(14.8) \quad 23(85.2) \quad 27(8.0)$

$35(12.1) \quad 254(87.9) \quad 289$

(85.3)

$15(30.0) \quad 35(70.0) \quad 50(14.7)$

$22(12.4) \quad 156(87.6) \quad 178$

(52.5)

$28(17.4) \quad 133(82.6)$

161

(47.5)

$145 \quad 104(527) \quad 106(623)$ (1621)

$25(28.4) \quad 63(71.6) \quad 88(26.0)$

$17(14.8) \quad 98(85.2) \quad 115$

(33.9)

$8(5.9) \quad 128(94.1)$

136

(40.1)
8 (8.3) $\quad 89(91.8) \quad 97(28.6)$

$18(9.9) \quad 164(90.1) \quad 182$

(53.7)

$23(41.1) \quad 33(58.9) \quad 56(16.5)$

$1(25.0) \quad 3(75.0) \quad 4(1.2)$

\section{History of defaults}


*Median (IQR); right skew; WHO World Health Organization; HIV human immunodeficiency virus; ART antiretroviral therapy; NRTI non-nucleoside reverse transcriptase

Table 3 Prognostic factors of survival among HIV-infected adults treated with ART in Hospital Sungai Buloh ( $n=339)$

\section{Variables}

\section{Crude \\ hazards ratio \\ $(95 \% \mathrm{Cl})$}

p-

Adjusted hazards ratio

p-

value

$(95 \% \mathrm{Cl})$

value

\section{Baseline haemoglobin level}

$(g / d L)$

No anaemia

1.00

$-$

1.00

Anaemia

$4.53(2.43, \quad<0.001$

$3.76(1.97,7.18)$

$<0.001$

$$
\text { 8.44) }
$$

Tuberculosis

No

1.00

$-$

1.00

Yes

$4.53(2.43, \quad<0.001$

$2.09(1.10,3.96)$

0.024

\subsection{4)}

\section{Figures}



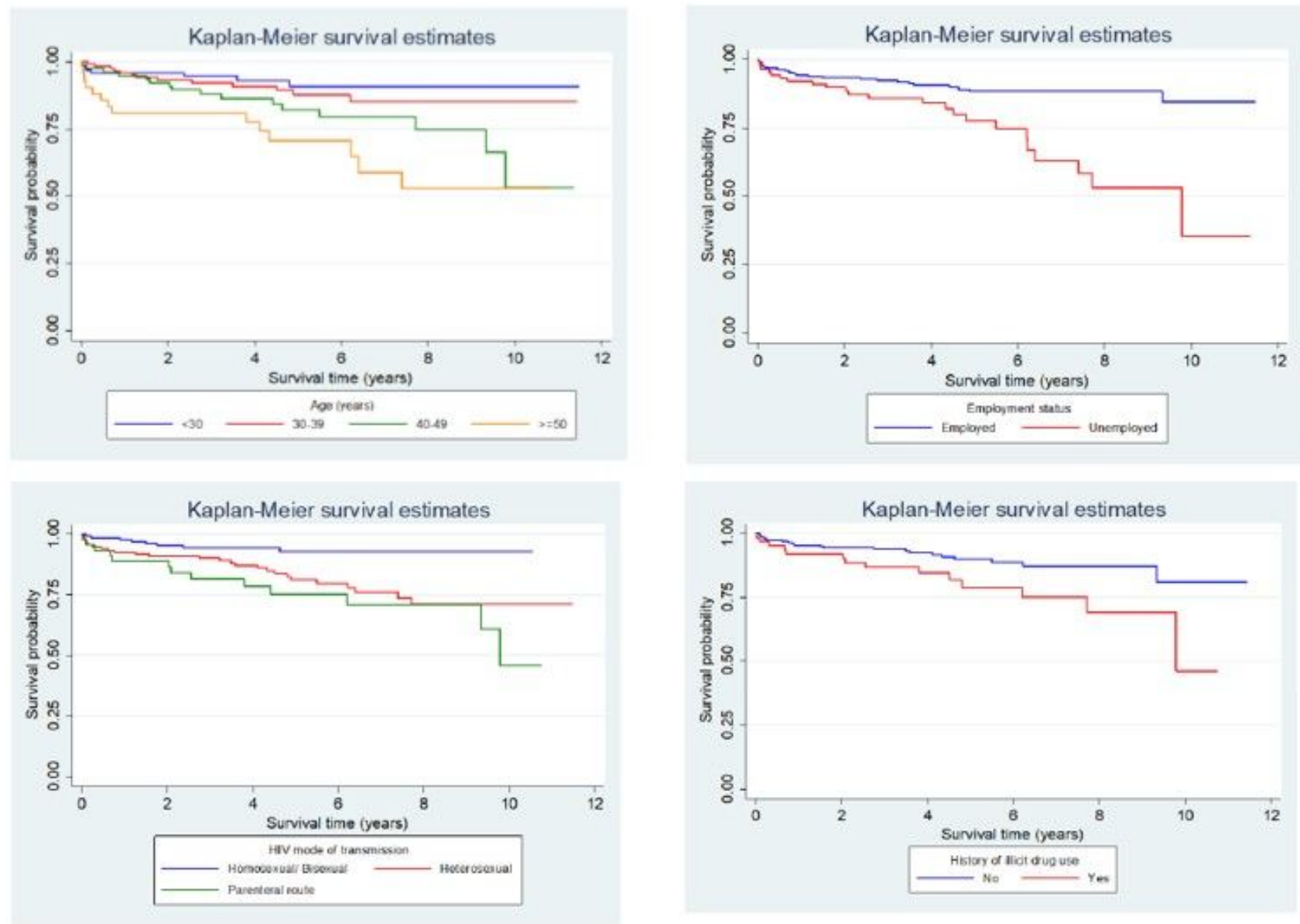

\section{Figure 1}

Kaplan-Meier estimates for overall survival rates of HIV-infected adults in Hospital Sungai Buloh based on age group; employment status; HIV transmission model; and history of illicit drug use. 

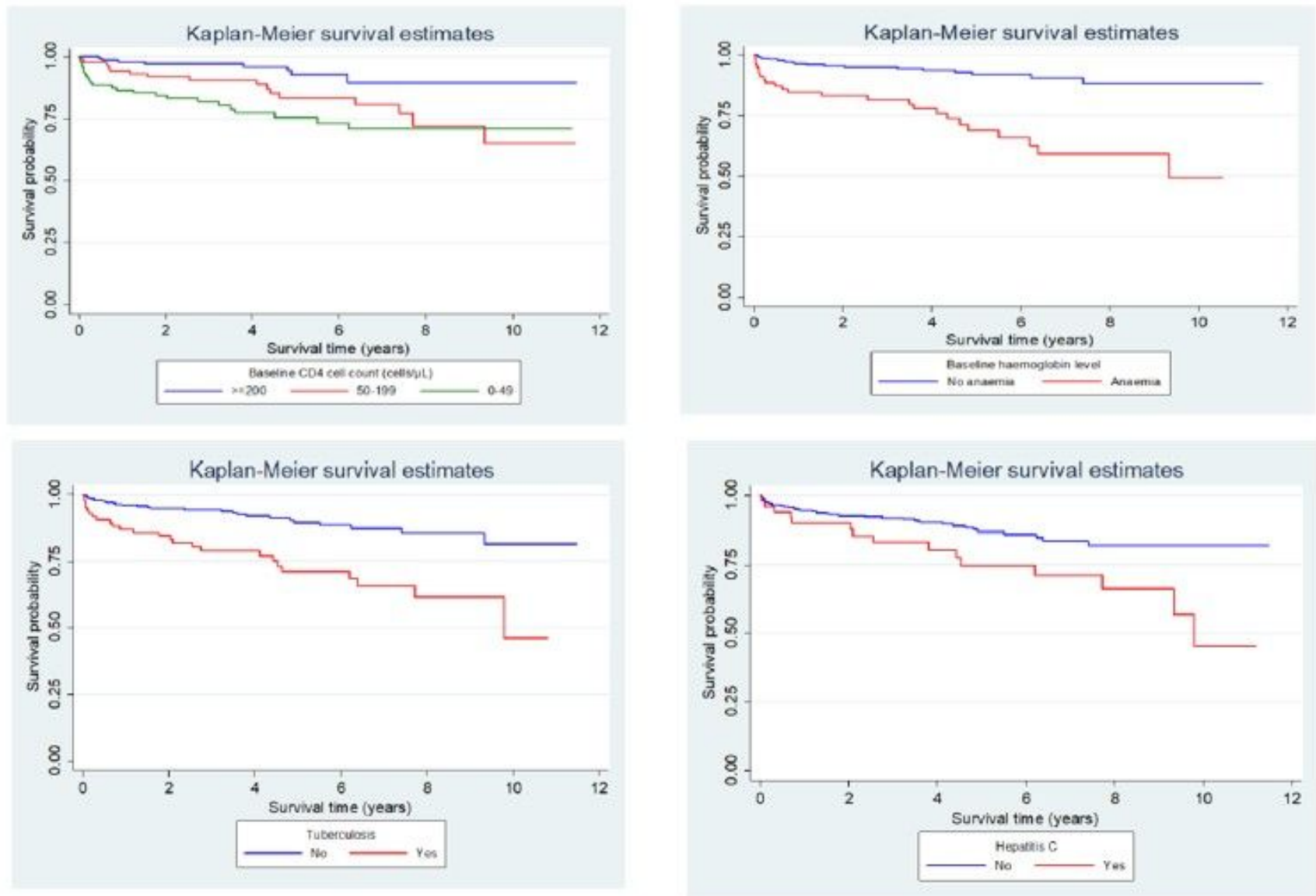

\section{Figure 2}

Kaplan-Meier estimates for overall survival rates of HIV-infected adults in Hospital Sungai Buloh based on baseline CD4 cell count; baseline haemoglobin level; tuberculosis; and hepatitis C.
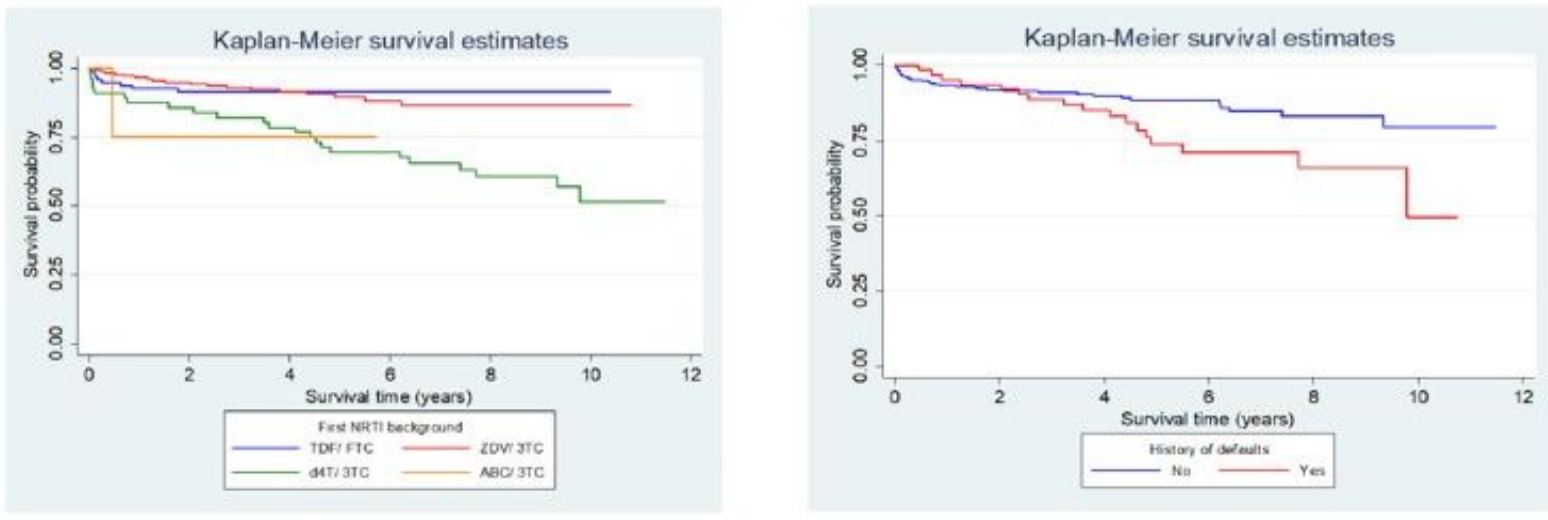

Figure 3 
Kaplan-Meier estimates for overall survival rates of HIV-infected adults in Hospital Sungai Buloh based on first NRTI background; and history of defaults. 